Research Article

\title{
Coiflet Wavelet-Homotopy Solution of Channel Flow due to Orthogonally Moving Porous Walls Governed by the Navier-Stokes Equations
}

\author{
Qing-Bo Chen and Hang Xu $\mathbb{D}$ \\ State Key Lab of Ocean Engineering, School of Naval Architecture, Ocean and Civil Engineering, Shanghai Jiao Tong University, \\ Shanghai 200240, China
}

Correspondence should be addressed to Hang Xu; hangxu@sjtu.edu.cn

Received 18 January 2020; Revised 1 April 2020; Accepted 10 April 2020; Published 15 May 2020

Academic Editor: Rafael López

Copyright ( 2020 Qing-Bo Chen and Hang Xu. This is an open access article distributed under the Creative Commons Attribution License, which permits unrestricted use, distribution, and reproduction in any medium, provided the original work is properly cited.

\begin{abstract}
A newly computational method based on the Coiflet wavelet and homotopy analysis method is developed, which inherits the great nonlinear treatment of the homotopy analysis technique and the local high-precision capability of the wavelet approach, to give solutions to the classic problem of channel flow with moving walls. The basic principle of this suggested technique and the specific solving process are presented in detail. Its validity and efficiency are then checked via rigid comparisons with other computational approaches. It is found that the homotopy-based convergence-control parameter and the wavelet-based resolution level of Coiflet are two effective ways to improve on accuracies of solutions.
\end{abstract}

\section{Introduction}

Liquid flow in porous channels has received extensive attention owing to its wide industrial and engineering applications. The earliest study on channel flow with porous walls was performed by Berman [1], who obtained a perturbation solution for small Reynolds number. Morduchow [2] formulated a closed-form solution of Berman's problem [1] over the full injection range by means of the method of averages. Berman's solution was then extended by Proudman [3] to the large Reynolds number case and further generalized by Wah [4] for both small and large Reynolds number cases. Other extended studies have been done by various researchers on different aspects such as the theoretical solution at high suction Reynolds number [5], experimental observation for injection and suction effects [6], exact solution at high suction or injection velocity [7], complete solution for large injection with the viscous layer effect [8], and high-precision series solution for the uniformly porous tube [9]. A significant extension was given by Brady and Acrivos [10] for inclusion of the accelerating walls into the classic channel flow problem. Watson et al. [11] further investigated the case that the channel walls undergo both acceleration and porosity. Dauenhauer and Majdalani [12] examined the unsteady channel flow caused by uniformly time-dependent wall expansion ratio in the presence of porous and moving walls. Their work was further extended by $\mathrm{Xu}$ et al. [13] to the nonuniform time-dependent wall expansion ratio case.

In fact, most of fluid flow problems are nonlinear problems, which bring great difficulties to give their solutions. Various analytical or numerical methods have been proposed to solve these nonlinear flow problems such as the Runge-Kutta method [14, 15], the Akbari-Ganji method (AGM) [16, 17], the homotopy perturbation method (HPM), the collocation method (CM), the finite element method (FEM) [18], the Galerkin finite element method (GFEM) [19], and other numerical methods [20, 21]. Homotopy method is widely used because of its superiority in solving nonlinear problems. At the same time, many homotopybased methods have also been proposed. Liu studied a parameter identification problem of two-phase porous 
media fractional flow equations [22] and then solved a nonlinear convection-diffusion equation [23] by a wavelet multiscale-homotopy method. In addition, based on the homotopy method, a multigrid-homotopy method was used to solve a nonlinear inverse problem governed by a convection-diffusion equation [24], and the numerical identification of diffusion parameters in a nonlinear convectiondiffusion equation was investigated by an adaptive homotopy perturbation method [25].

Recently, the homotopy analysis method (HAM) [26] was developed for handling strong nonlinear problems, which possesses several advantages such as freedom to choose solution expressions, independence of small or large parameters, and adjustment of solution convergence. It has been used to solve many kinds of nonlinear problems in different fields $[27,28]$. However, solutions by this technique are purely based on analytical computation; as a result, it is very time consuming when nonlinear problems are very complicated. On the contrary, the Coiflet wavelet technique [29] received applausive attention owing to its excellent features including feasible wavelet transformation, high computational efficiency, and superior expression of frequency and time information. This technique was initially used in signal analysis [30] and recently extended by Zhou et al. [31, 32] and Liu et al. [33, 34] to some classic nonlinear homogeneous problems in applied mathematics and mechanics. Very recently, the homotopy-based Coiflet wavelet method was developed by Yang and Liao [35, 36] for capturing solutions with high precision to nonlinear equations. One-dimensional and two-dimensional Bratus equations subjected to homogeneous boundary conditions were considered in their work as illustrative examples. Their proposed technique was then extended by $\mathrm{Yu}$ and his collaborators to solve the nonlinear plate bending problem [37] and cavity flow problem [38] with nonhomogeneous boundary conditions.

Though several successful applications of the homotopyCoiflet wavelet technique have been achieved, many aspects of this approach are not yet clear. Further efforts are still needed to improve on its computational capability and efficiency. The aim of this paper is to extend the homotopyCoiflet wavelet method to solve the classic porous channel flow problem governed by a fourth-order strong nonlinear system subjected to nonhomogeneous boundary conditions. The basic idea of this approach will be exhibited in detail. A range of wall expansion ratios for both injection and suction cases, especially for large injection and suction, will be taken into account. The accuracy and efficiency of our results will be then checked via a rigid comparison with previous studies.

\section{Mathematical Description}

As shown in Figure 1, liquid flow is driven through a twodimensional rectangular channel bounded by a porous wall at $y= \pm a$. The channel walls undergo uniform expansion or contraction. The constant ratio of fluid injection and suction through the two opposing channel walls is applied. The fixed Cartesian coordinates $(x, y)$ in the space are used,

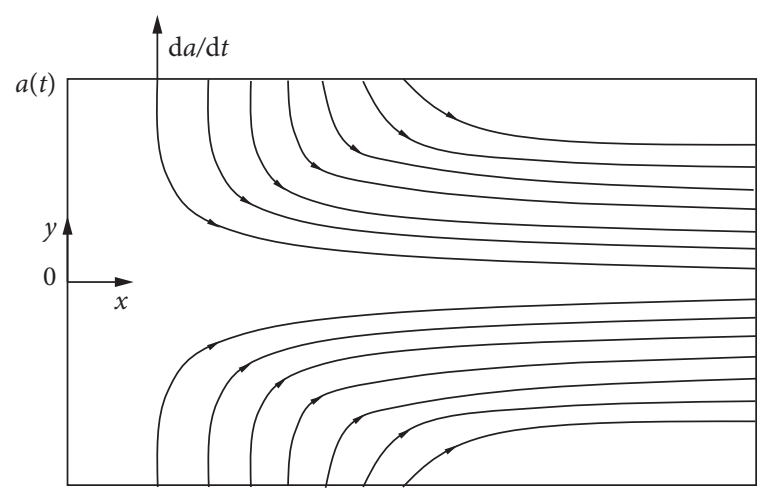

Figure 1: Physical sketch.

with the $x$-axis extending along the length of the channel and the $y$-axis in the wall-normal direction.

The fourth-order nonlinear system describing fluid flow in this porous channel with moving walls is written as (refer to Dauenhauer and Majdalani [12])

$$
F^{\prime \prime \prime \prime}+F F^{\prime \prime \prime}+\left(3 \alpha-F^{\prime}\right) F^{\prime \prime}+\alpha \eta F^{\prime \prime \prime}=0,
$$

subject to the boundary conditions

$$
\begin{aligned}
F(0) & =0, \\
F^{\prime \prime}(0) & =0, \\
F(1) & =R, \\
F^{\prime}(1) & =0,
\end{aligned}
$$

where prime denotes the derivation with $\eta(\eta=y / a), F$ is the dimensionless stream function and the function of $\eta, \alpha$ is the wall expansion and extraction ratio, and $R$ is the Reynolds number with positive $R$ representing the injection case and vice versa. Note that this equation is derived directly from the Navier-Stokes equation via scaling and vorticity transformations without reduction. As a result, its solution can be deemed as the exact one of this famous equation.

2.1. Linearization. Here, the homotopy analysis method is employed to linearize nonlinear equation (1) and its boundary conditions (2). In doing so, we construct the following zeroth-order deformation equation:

$$
(1-q) \mathscr{L}\left[\phi(\eta ; q)-F_{0}(\eta)\right]=c_{0} q \mathcal{N}[\phi(\eta ; q)],
$$

subject to the boundary conditions

$$
\begin{aligned}
& \phi(0 ; q)=0,\left.\quad \frac{\partial^{2} \phi(\eta ; q)}{\partial \eta^{2}}\right|_{\eta=0}=0, \\
& \phi(1 ; q)=R,\left.\quad \frac{\partial \phi(\eta ; q)}{\partial \eta}\right|_{\eta=1}=0,
\end{aligned}
$$

where $q$ is an embedding parameter evolving from 0 to $1, \mathscr{L}$ is the auxiliary linear operator, $F_{0}(\eta)$ is the initial guess that satisfies all given boundary conditions, $c_{0}$ is the convergence-control parameter, $\phi(\eta ; q)$ is the mapping function of 
$F(\eta)$, and $\mathcal{N}[\phi(\eta ; q)]$ is the nonlinear operator, which is defined by

$$
\begin{aligned}
\mathcal{N}[\phi(\eta ; q)]= & \frac{\partial^{4} \phi(\eta ; q)}{\partial \eta^{4}}+\phi(\eta ; q) \frac{\partial^{3} \phi(\eta ; q)}{\partial \eta^{3}}+3 \alpha \frac{\partial^{2} \phi(\eta ; q)}{\partial \eta^{2}} \\
& -\frac{\partial \phi(\eta ; q)}{\partial \eta} \frac{\partial^{2} \phi(\eta ; q)}{\partial \eta^{2}}+\alpha \eta \frac{\partial^{3} \phi(\eta ; q)}{\partial \eta^{3}} .
\end{aligned}
$$

Since the computational domain is $[0,1]$, the highestorder derivative of equation (1) is fourth-order, and the quantity of boundary conditions is four; we choose $\mathscr{L}[\phi(x ; q)]=\partial^{4} \phi(x ; q) / \partial x^{4}$ as the auxiliary linear operator for this problem.

From equation (3), it is readily known $\phi(\eta ; 0)=F_{0}(\eta)$ and $\phi(\eta ; 1)=F(\eta)$. To get the exact solution $F(\eta)$, we expand the solution $\phi(\eta ; q)$ of equation (3) by

$$
\phi(\eta ; q)=F_{0}(\eta)+\sum_{m=1}^{+\infty} F_{m}(\eta) q^{m}
$$

where $F_{m}(\eta)$ is the high-order subfunction. Clearly, $F(\eta)=$ $\phi(\eta ; 1)$ is acquired at $q=1$.

Note that the initial guess should satisfy the given boundary conditions. According to equation (4), the initial guess $F_{0}(\eta)$ is chosen as

$$
F_{0}(\eta)=\frac{1}{2} R \eta\left(3-\eta^{2}\right) .
$$

To determine $F_{m}(\eta)$, we construct the high-order deformation equation, by differentiating equation (3) $m$ times with respect to $q$, then dividing by $m$ !, and finally setting $q=0$, as

$$
\mathscr{L}\left[F_{m}(\eta)-\chi_{m} F_{m-1}(\eta)\right]=c_{0} R_{m}\left[\vec{F}_{m-1}(\eta)\right],
$$

where

$$
\begin{aligned}
\chi_{m} & = \begin{cases}0, & m=1, \\
1, & m>1\end{cases} \\
F_{m}(\eta) & =\left.\frac{1}{m !} \frac{\partial^{m} \phi(\eta ; q)}{\partial q^{m}}\right|_{q=0}, \\
R_{m}\left[\vec{F}_{m-1}(\eta)\right] & =\left.\frac{1}{(m-1) !} \frac{\partial^{m-1} \mathcal{N}[\phi(\eta ; q)]}{\partial q^{m-1}}\right|_{q=0},
\end{aligned}
$$

in which $\vec{F}_{m-1}(\eta)$ denotes a group of subfunctions $F_{0}(\eta), F_{1}(\eta), F_{2}(\eta), \ldots, F_{m-1}(\eta)$.

The corresponding high-order boundary conditions then take the form

$$
\begin{aligned}
& F_{m}(0)=0, \\
& F_{m}^{\prime \prime}(0)=0, \\
& F_{m}(1)=0, \\
& F_{m}^{\prime}(1)=0 .
\end{aligned}
$$

2.2. Coiflet Wavelet Expression. Different from the pure HAM technique, we select the Coiflet wavelet function rather than the power series given by $\mathrm{Xu}$ et al. [13] as the solution expression of $F(\eta)$.

Following Wang [39] and Liu [40], an arbitrary function $f(x)$ is approximated by Coiflet wavelet as

$$
f(x) \approx \sum_{k=2-3 N+M_{1}}^{2^{j}-1+M_{1}} f\left(\frac{k}{2^{j}}\right) \varphi\left(2^{j} x-k+M_{1}\right),
$$

where $N$ is the vanishing moment, $M_{1}$ is the first-order vanishing moment, and $\varphi$ is the wavelet basis.

In practical applications of wavelet approximation in boundary value problems, boundary modification is needed for handling boundary conditions. The modified boundary technique $[32,34,39]$ for Coiflet wavelet is constructed as

$$
\varphi_{j, k}(x)= \begin{cases}\sum_{i=2-3 N+M_{1}}^{-1} T_{0, k}\left(\frac{i}{2^{j}}\right) \varphi_{j, i}+\varphi_{j, k}, & k \in[0,3], \\ \varphi_{j, k}, & k \in\left[4,2^{j}-4\right], \\ \sum_{i=2^{j}+1}^{j-1+M_{1}} T_{1,2^{j}-k}\left(\frac{i}{2^{j}}\right) \varphi_{j, i}+\varphi_{j, k}, & k \in\left[2^{j}-3,2^{j}\right],\end{cases}
$$

in which

$$
\begin{aligned}
\varphi_{j, i} & =\phi\left(2^{j} x-i+M_{1}\right), \\
\varphi_{j, k} & =\phi\left(2^{j} x-k+M_{1}\right), \\
T_{0, k}(x) & =\sum_{i=0}^{3} \frac{p_{0, i, k}}{i !} x^{i},
\end{aligned}
$$

$$
T_{1, k}(x)=\sum_{i=0}^{3} \frac{p_{1, i, k}}{i !}(x-1)^{i}
$$

Note that $p_{0, i, k}$ and $p_{1, i, k}$ are coefficients determined by the following coefficient matrices $P_{0}$ and $P_{1}$

$$
P_{0}=\left[\begin{array}{cccc}
1 & 0 & 0 & 0 \\
-\left(\frac{11}{6}\right) & 3 & -\left(\frac{3}{2}\right) & \frac{1}{3} \\
2 & -5 & 4 & -1 \\
-1 & 3 & -3 & 1
\end{array}\right],
$$

$$
P_{1}=\left[\begin{array}{cccc}
1 & 0 & 0 & 0 \\
\frac{11}{6} & -3 & \frac{3}{2} & -\left(\frac{1}{3}\right) \\
2 & -5 & 4 & -1 \\
1 & -3 & 3 & -1
\end{array}\right],
$$


via the relation $P_{0}=\left\{2^{-i j} p_{0, i, k}\right\}$ and $P_{1}=\left\{2^{-i j} p_{1, i, k}\right\}$ with $i=0,1,2,3$. Taking the boundary conditions (4) into consideration, it is known that $F(\eta)$ satisfies the inhomogeneous Dirichlet and homogeneous Neumann types of boundary conditions. Hence, following $\mathrm{Yu}$ et al. [37, 38, 41-43], by modifying the coefficient matrices, the Coiflet wavelet basis is selected as

$$
h_{j, k}(\eta)=\left.\varphi_{j, k}(\eta)\right|_{p_{0,2, k} \longrightarrow 0, p_{1,1, k} \longrightarrow 0^{\circ}}
$$

Furthermore, $F_{m}(\eta)$ and $R_{m}(\eta)$ can be expressed by Coiflet wavelet as

$$
\begin{aligned}
F_{m}(\eta) & \approx \sum_{k=0}^{2^{j}} F_{m}\left(\frac{k}{2^{j}}\right) h_{j, k}(\eta)=\sum_{k=1}^{2^{j}-1} F_{m}\left(\frac{k}{2^{j}}\right) h_{j, k}(\eta) \\
R_{m}\left[\vec{F}_{m-1}(\eta)\right] & \approx \sum_{k=0}^{2^{j}} R_{m}\left(\frac{k}{2^{j}}\right) \varphi_{j, k}(\eta),
\end{aligned}
$$

where

$$
\begin{aligned}
R_{m}\left(\frac{k}{2^{j}}\right)= & F_{m-1}^{\prime \prime \prime \prime}\left(\frac{k}{2^{j}}\right)+3 \alpha F_{m-1}^{\prime \prime}\left(\frac{k}{2^{j}}\right)+\alpha \eta F_{m-1}^{\prime \prime \prime}\left(\frac{k}{2^{j}}\right) \\
& +\sum_{s=0}^{m-1}\left\{F_{s}\left(\frac{k}{2^{j}}\right) F_{m-1-s}^{\prime \prime \prime}\left(\frac{k}{2^{j}}\right)-F_{s}^{\prime}\left(\frac{k}{2^{j}}\right) F_{m-1-s}^{\prime \prime}\left(\frac{k}{2^{j}}\right)\right\} .
\end{aligned}
$$

Note that the boundary conditions on both sides of the Coiflet wavelet expansion of $F_{m}(\eta)$ are rectified due to the boundary condition constraints at $\eta=0$ and $\eta=1$.

Generally, from Coiflet wavelet approximation (16), the $n$ th-order derivatives with respect to $\eta$ are obtained by the following formula as

$$
\frac{\mathrm{d}^{n} F_{m}(\eta)}{\mathrm{d} \eta^{n}} \approx \sum_{k=1}^{2^{j}-1} F_{m}\left(\frac{k}{2^{j}}\right) \frac{\mathrm{d}^{n} h_{j, k}(\eta)}{\mathrm{d} \eta^{n}} .
$$

Therefore, $m$ th-order deformation equation (8) is expressed as

$$
\begin{aligned}
& \sum_{k=1}^{2^{j}-1}\left[F_{m}\left(\frac{k}{2^{j}}\right)-\chi_{m} F_{m-1}\left(\frac{k}{2^{j}}\right)\right] \mathscr{L}\left[h_{j, k}(\eta)\right] \\
& \quad=c_{0} \sum_{k=0}^{2^{j}} R_{m}\left(\frac{k}{2^{j}}\right) \varphi_{j, k}(\eta) .
\end{aligned}
$$

Following Yang and Liao [35, 36], the Galerkin method is employed to solve $m$ th-order deformation equation (19). Multiplying both sides of equation (19) by $h_{j, l}(\eta)(l=$ $\left.1,2,3, \ldots, 2^{j}-1\right)$ and then integrating on the domain $[0,1]$, we obtain the $m$ th-order wavelet-Galerkin deformation equation as

$$
\mathbf{A}^{\mathrm{T}}\left(\widehat{\mathbf{F}}_{\mathbf{m}}-\chi_{m} \widehat{\mathbf{F}}_{\mathbf{m}-\mathbf{1}}\right)=c_{0} \mathbf{B}^{\mathrm{T}} \widehat{\mathbf{R}}_{m},
$$

where

$$
\begin{aligned}
& \widehat{\mathbf{F}}_{\mathbf{m}}=\left\{F_{m}\left(\frac{1}{2^{j}}\right), F_{m}\left(\frac{2}{2^{j}}\right), \ldots, F_{m}\left(\frac{2^{j}-1}{2^{j}}\right)\right\}^{T}, \\
& \widehat{\mathbf{R}}_{\mathbf{m}}=\left\{R_{m}\left(\frac{0}{2^{j}}\right), R_{m}\left(\frac{1}{2^{j}}\right), \ldots, R_{m}\left(\frac{2^{j}}{2^{j}}\right)\right\}^{T},
\end{aligned}
$$

and the connection coefficient matrices are defined as

$$
\begin{aligned}
& \mathbf{A}=\left\{a_{k, l}=\Gamma_{\mathbf{k}, 1}^{\mathrm{j}, 4}\right\}_{k, l=1}^{k, l=2^{j}-1}, \\
& \mathbf{B}=\left\{b_{k, l}=\bar{\Gamma}_{\mathrm{k}, 1}^{\mathrm{j}, 0}\right\}_{k=0, l=1}^{k=2^{j}, l=2^{j}-1},
\end{aligned}
$$

in which $\Gamma_{k, l}^{j, n}$ and $\bar{\Gamma}_{k, l}^{j, n}$ are the generalized connection coefficients defined by

$$
\begin{aligned}
\Gamma_{k, 1}^{j, n} & =\int_{0}^{1} \frac{\mathrm{d}^{n} h_{j, k}(\xi)}{\mathrm{d} x^{n}} h_{j, l}(\xi) \mathrm{d} \xi, \\
\bar{\Gamma}_{\mathrm{k}, 1}^{j, n} & =\int_{0}^{1} \frac{\mathrm{d}^{n} \varphi_{j, k}(\xi)}{\mathrm{d} x^{n}} h_{j, l}(\xi) \mathrm{d} \xi .
\end{aligned}
$$

Note that the generalized connection coefficients change as the auxiliary linear operator $\mathscr{L}$ and boundary conditions vary.

By solving the $m$ th-order wavelet-Galerlin equations from the order $m=1,2, \ldots$, the wavelet homotopy solution can be completely determined.

\section{Validation}

3.1. Validation in the PDE Case. In this section, we will present a $2 \mathrm{D}$ linear problem to verify the accuracy and efficiency of our proposed method. The governing equation considered here is as follows:

$$
\frac{\partial^{2} f}{\partial x^{2}}+\frac{\partial^{2} f}{\partial y^{2}}+2 \pi^{2} f_{1}=0
$$

where

$$
f_{1}(x, y)=\cos (\pi x) \cos (\pi y) .
$$

The corresponding homogeneous Neumann boundary conditions are

$$
\begin{aligned}
& \left.\frac{\partial f}{\partial x}\right|_{x=0}=0, \\
& \left.\frac{\partial f}{\partial x}\right|_{x=1}=0, \\
& \left.\frac{\partial f}{\partial y}\right|_{y=0}=0, \\
& \left.\frac{\partial f}{\partial y}\right|_{y=1}=0 .
\end{aligned}
$$

Note that equation (24) has the analytical solution

$$
f_{e}(x, y)=\cos (\pi x) \cos (\pi y) .
$$


In our approach, by modifying the coefficient matrices, the Coiflet wavelet basis is expressed by

$$
\begin{gathered}
h_{j, k}(x)=\left.\varphi_{j, k}(x)\right|_{p_{0,1, i} \longrightarrow 0, p_{1,1, i} \longrightarrow 0^{\prime}}, \\
h_{j, l}(y)=\left.\varphi_{j, l}(y)\right|_{p_{0,1, i} \longrightarrow 0, p_{1,1, i} \longrightarrow 0} .
\end{gathered}
$$

Furthermore, $f(x, y)$ can be expressed by Coiflet wavelet as

$$
\begin{gathered}
f(x, y) \approx \sum_{k=0}^{2^{j}} \sum_{l=0}^{2^{j}} f\left(\frac{k}{2^{j}}, \frac{l}{2^{j}}\right) h_{j, k}(x) h_{j, l}(y), \\
f_{1}(x, y) \approx \sum_{k=0}^{2^{j}} \sum_{l=0}^{2^{j}} f\left(\frac{k}{2^{j}}, \frac{l}{2^{j}}\right) \varphi_{j, k}(x) \varphi_{j, l}(y) .
\end{gathered}
$$

Therefore, equation (24) is expressed as

$$
\begin{aligned}
& \sum_{k=0}^{2^{j}} \sum_{l=0}^{2^{j}} f\left(\frac{k}{2^{j}}, \frac{l}{2^{j}}\right) \frac{\mathrm{d}^{2} h_{j, k}(x)}{\mathrm{d} x^{2}} h_{j, l}(y) \\
& +\sum_{k=0}^{2^{j}} \sum_{l=0}^{2^{j}} f\left(\frac{k}{2^{j}}, \frac{l}{2^{j}}\right) h_{j, k}(x) \frac{\mathrm{d}^{2} h_{j, l}(y)}{\mathrm{d} y^{2}} \\
& +2 \pi^{2} \sum_{k=0}^{2^{j}} \sum_{l=0}^{2^{j}} f_{1}\left(\frac{k}{2^{j}}, \frac{l}{2^{j}}\right) \varphi_{j, k}(x) \varphi_{j, l}(y)=0 .
\end{aligned}
$$

As described above, multiplying both sides of equation (30) by $h_{j, n}(x) h_{j, m}(y)\left(n, m=0 \sim 2^{j}\right)$ and then applying the multiple integrals on the domain $[0,1]^{2}$, we obtain the wavelet-Galerkin equation as

$$
\left(\widetilde{\mathbf{A}}_{1}^{T}+{\widetilde{\mathbf{A}_{2}}}^{T}\right) \cdot \operatorname{vecf}+\widetilde{\mathbf{B}}^{T} \cdot \operatorname{vec} \mathbf{f}_{1}=0,
$$

where $\operatorname{vec}(\cdot)$ is a straighten vector operator which pulls a matrix into a vector row by row and $\mathbf{f}, \mathbf{f}_{1}$ is a $\left(2^{j}+1\right) \times$ $\left(2^{j}+1\right)$ matrix defined by

$$
\begin{aligned}
\mathbf{f} & =\left\{f_{k, l}=f\left(\frac{k}{2^{j}}, \frac{l}{2^{j}}\right)\right\}, \\
\mathbf{f}_{1} & =\left\{f_{k, l}^{1}=f_{1}\left(\frac{k}{2^{j}}, \frac{l}{2^{j}}\right)\right\},
\end{aligned}
$$

where the coefficient matrices are obtained through

$$
\left\{\begin{array}{l}
\widetilde{\mathbf{A}_{1}}=\Gamma_{k, n}^{j, 2} \otimes \Gamma_{l, m}^{j, 0}, \\
\widetilde{\mathbf{A}_{2}}=\Gamma_{k, n}^{j, 0} \otimes \Gamma_{l, m}^{j, 2}, \\
\widetilde{\mathbf{B}}=2 \pi^{2} \cdot \bar{\Gamma}_{k, n}^{j, 0} \otimes \bar{\Gamma}_{l, m}^{j, 0} .
\end{array}\right.
$$

Here, $\otimes$ denotes the operator of the Kronecker tensor product, $\widetilde{\mathbf{A}_{1}}, \widetilde{\mathbf{A}_{2}}$, and $\widetilde{\mathbf{B}}$ are $\left(2^{j}+1\right) \times\left(2^{j}+1\right)$ matrices, and the generalized connection coefficients $\Gamma$ and $\bar{\Gamma}$ are defined as equation (23).

To illustrate the error level of our solution, we define the average square error ErrSQ based on the exact solution (27) as follows:

$$
\operatorname{ErrSQ}=\frac{1}{\left(2^{j}+1\right)^{2}} \sum_{k=0}^{2^{j}} \sum_{l=0}^{2^{j}}\left[f\left(\frac{k}{2^{j}}, \frac{l}{2^{j}}\right)-f_{e}\left(\frac{k}{2^{j}}, \frac{l}{2^{j}}\right)\right]^{2} .
$$

The solution of equation (24) is shown in Figure 2, whose residual error is listed in Table 1 . It is found that the average square error decreases rapidly with the resolution level $j$ increasing. For $j=7$, especially, the error reaches to $10^{-9}$ within 1 minute CPU time. This completely demonstrates the accuracy and great efficiency of our proposed method.

3.2. Convergence and Error Analysis in the Nonlinear Case. To further verify the accuracy of our proposed method on nonlinear problems, we will calculate a special case of the fourth-order nonlinear system describing fluid flow in this porous channel and compare it with other numerical results.

Firstly, to test the convergence of our solution, we define the following residual error function:

$$
\operatorname{ResErr}_{F}=\frac{1}{2^{j}+1} \sum_{k=0}^{2^{j}}\left[\bar{F}_{M}\left(\frac{k}{2^{j}}\right)-\bar{F}_{M-1}\left(\frac{k}{2^{j}}\right)\right]^{2},
$$

where $\bar{F}_{M}$ and $\bar{F}_{M-1}$ denote the $m$ th-order and the $m-1$ th-order wavelet solutions, respectively.

Then, we used the numerical solution by means of the software package BVP4c in software Matlab as a reference solution $F_{\text {ref }}$ since the original problem did not have an exact analytical solution. Therefore, we define the relative error as follows:

$$
\operatorname{Err}_{M}=\frac{1}{2^{j}+1} \sum_{k=0}^{2^{j}}\left[\bar{F}_{M}\left(\frac{k}{2^{j}}\right)-F_{\mathrm{ref}}\left(\frac{k}{2^{j}}\right)\right]^{2}
$$

Based on the definitions of Dauenhauer and Majdalani [12], the axial velocity $U$ and the normal velocity $V$ are given by

$$
\begin{aligned}
& U=\frac{F^{\prime}}{R}, \\
& V=\frac{F}{R} .
\end{aligned}
$$

The residual error functions of the axial and normal velocities are therefore expressed as

$$
\begin{aligned}
& \operatorname{ResErr}_{U}=\frac{1}{2^{j}+1} \sum_{k=0}^{2^{j}}\left[\bar{U}_{M}\left(\frac{k}{2^{j}}\right)-\bar{U}_{M-1}\left(\frac{k}{2^{j}}\right)\right]^{2}, \\
& \operatorname{ResErr}_{V}=\frac{1}{2^{j}+1} \sum_{k=0}^{2^{j}}\left[\bar{V}_{M}\left(\frac{k}{2^{j}}\right)-\bar{V}_{M-1}\left(\frac{k}{2^{j}}\right)\right]^{2} .
\end{aligned}
$$

We start our verification for $\alpha=0$, corresponding to the case of fluid flow through the porous channel with fixed walls. The injection Reynolds number is chosen as $R=5$, and the convergence-control parameters for $U$ and $V$ are designated as $c_{0}=-0.08$ and $c_{0}=-0.05$, respectively. As shown in Figure 3, the residual errors for both $U$ and $V$ reduce very rapidly as the computational order evolves. The least residual 


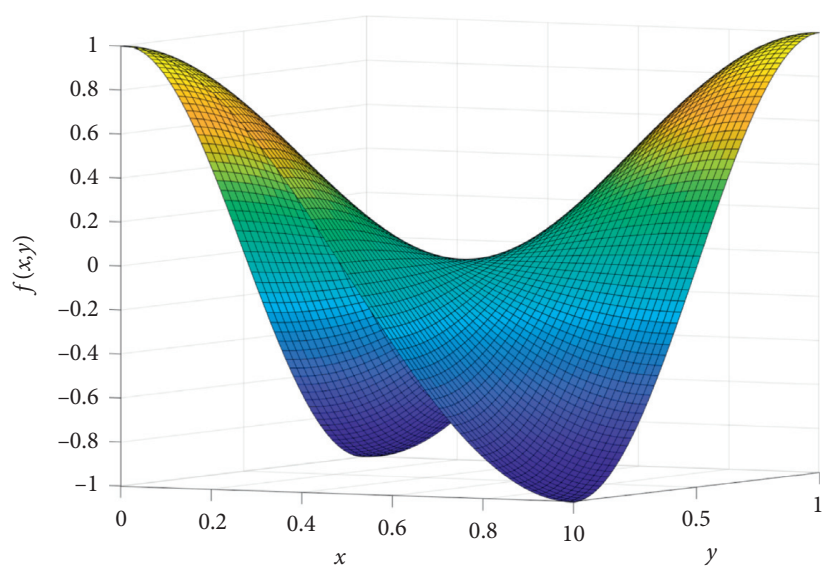

FiguRE 2: The wavelet solution of our example problem with $j=6$.

TABLE 1: The computational time and average square error ErrSQ for equation (24).

\begin{tabular}{lcccc}
\hline Resolution level & $j=4$ & $j=5$ & $j=6$ & $j=7$ \\
\hline CPU time (sec.) & 0.4349 & 0.6384 & 1.7153 & 32.3233 \\
ErrSQ & 2754.2368 & 0.35971 & $4.4064 \times 10^{-5}$ & $4.4154 \times 10^{-9}$ \\
\hline
\end{tabular}

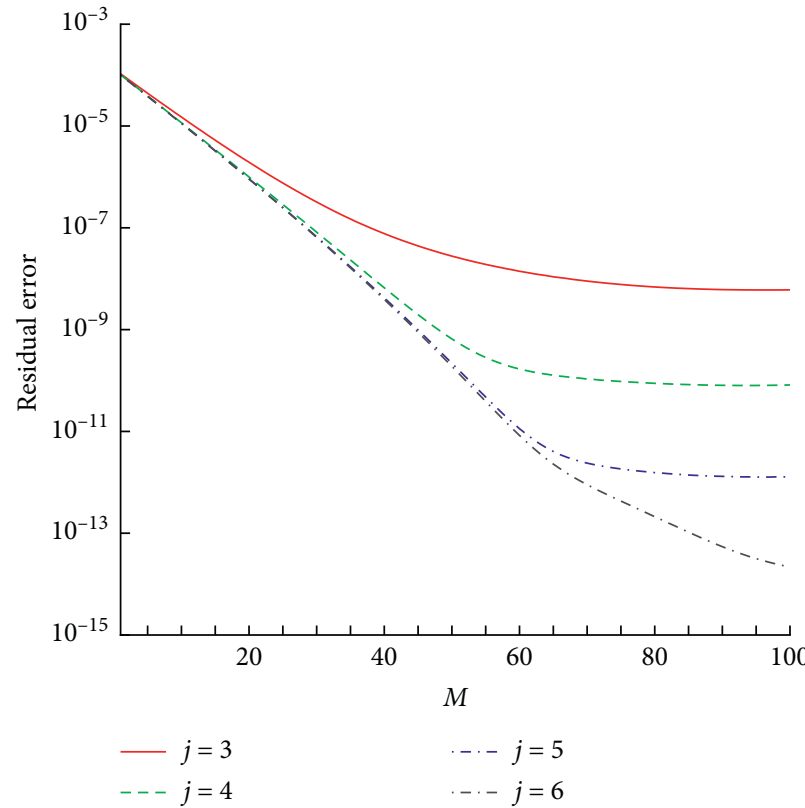

(a)

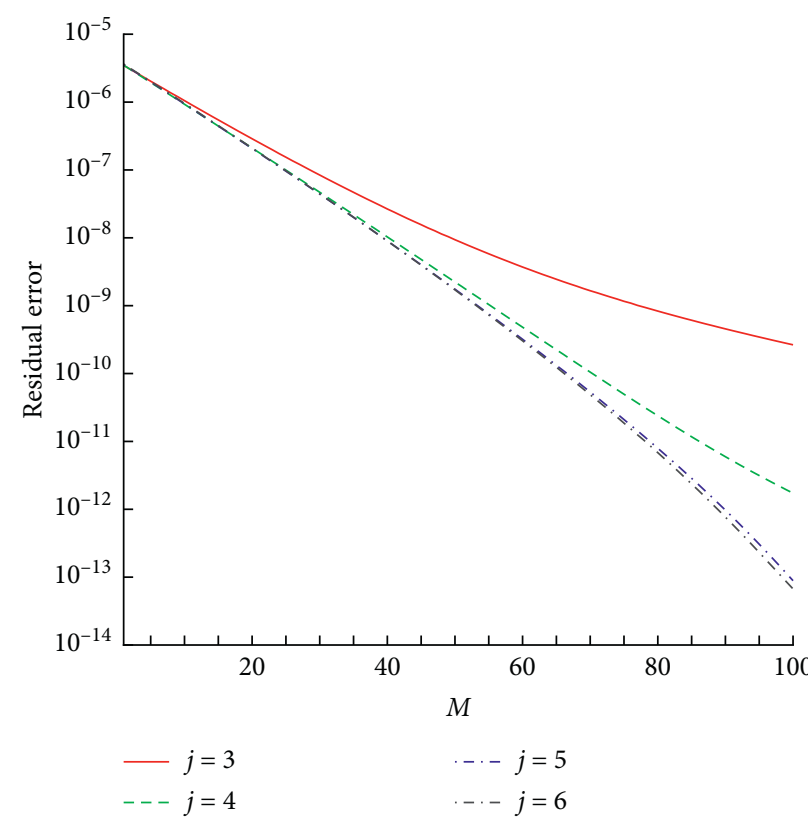

(b)

Figure 3: Residual errors at different resolution levels with $c_{0}=-0.08$ (for $U$ ) and $c_{0}=-0.05$ (for $V$ ). (a) Residual error for $U$. (b) Residual error for $V$.

error reaches about $10^{-14}$ at the computational order $M=$ 100 when the resolution level $j=6$. This figure clearly shows that error reduction can be achieved either by the increment of the resolution level or the increase of the computational order.

We then check results of the axial velocity $U$ and the normal velocity $V$ for different prescribed points at certain computational orders. As shown in Table 2 and Table 3, the computational results at each discrete point approach to the fixed values very quickly. Furthermore, we compare these results with the numerical ones, and the very excellent agreement is found, as shown in Figure 4. The corresponding computational time and the relative error for different resolution levels are listed in Table 4 . It is seen that the computational efficiency is high enough to give highly accurate solutions in one second, and our wavelet solution is very close to the numerical result, and the relative error can reach to $10^{-11}$. Note that the wavelet-homotopy 
TABLe 2: Different computational order results of the axial velocity $U$ at $R=5$ and $\alpha=0$ in the case of $j=6$ and $c_{0}=-0.08$.

\begin{tabular}{lcccccc}
\hline$\eta$ & $M=1$ & $M=10$ & $M=20$ & $M=50$ & $M=80$ & $M=100$ \\
\hline 0 & 1.502858 & 1.517773 & 1.523103 & 1.525142 & 1.525168 & 1.499865 \\
0.125 & 1.479219 & 1.493060 & 1.497980 & 1.499843 & 1.423985 & 1.499864 \\
0.250 & 1.408306 & 1.418936 & 1.422638 & 1.423976 & 1.297798 & 1.223984 \\
0.375 & 1.290140 & 1.295552 & 1.297294 & 1.297806 & 1.122277 & 0.899666 \\
0.500 & 1.124799 & 1.123476 & 1.122758 & 0.899685 & 0.633978 \\
0.625 & 0.912493 & 0.904120 & 0.900979 & 0.633983 & 0.331314 & 0.633979 \\
0.750 & 0.653655 & 0.640257 & 0.635619 & 0.331306 & 0.331315 \\
0.875 & 0.349064 & 0.336540 & 0.332519 & 0 & 0 \\
1 & 0 & 0 & 0 & & 0 \\
\hline
\end{tabular}

TABLE 3: Different computational order results of the normal velocity $V$ at $R=5$ and $\alpha=0$ in the case of $j=6$ and $c_{0}=-0.05$.

\begin{tabular}{|c|c|c|c|c|c|c|}
\hline$\eta$ & $M=1$ & $M=10$ & $M=20$ & $M=50$ & $M=80$ & $M=100$ \\
\hline 0 & 0 & 0 & 0 & 0 & 0 & 0 \\
\hline 0.125 & 0.186741 & 0.188134 & 0.188910 & 0.189531 & 0.189588 & 0.189591 \\
\hline 0.250 & 0.367592 & 0.370173 & 0.371609 & 0.372749 & 0.372852 & 0.372858 \\
\hline 0.375 & 0.536662 & 0.540030 & 0.541894 & 0.543357 & 0.543484 & 0.543491 \\
\hline 0.500 & 0.688065 & 0.691646 & 0.693610 & 0.695122 & 0.695246 & 0.695251 \\
\hline 0.625 & 0.815925 & 0.819047 & 0.820735 & 0.822000 & 0.822094 & 0.822096 \\
\hline 0.750 & 0.914391 & 0.916444 & 0.917534 & 0.918319 & 0.918370 & 0.918370 \\
\hline 0.875 & 0.977658 & 0.978392 & 0.978772 & 0.979033 & 0.979047 & 0.979047 \\
\hline 1 & 1 & 1 & 1 & 1 & 1 & 1 \\
\hline
\end{tabular}

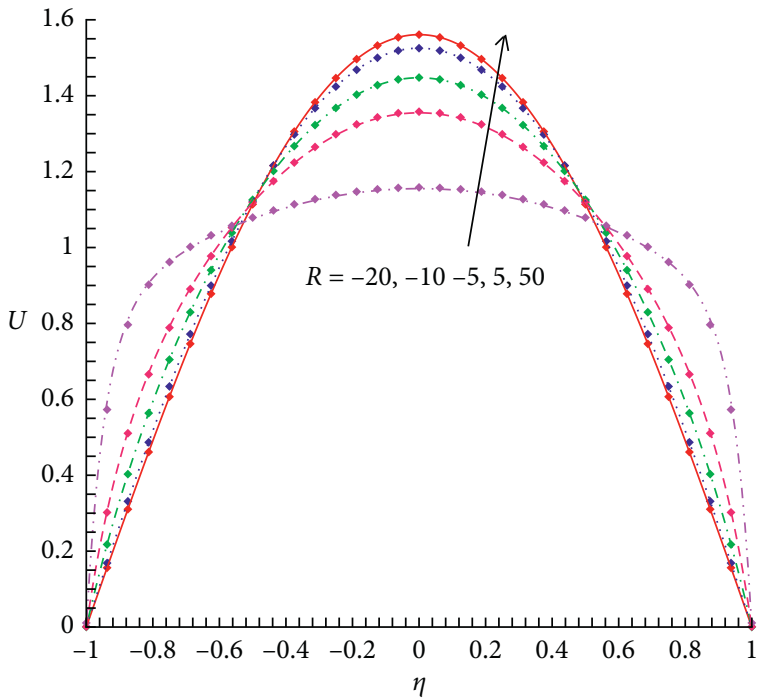

(a)

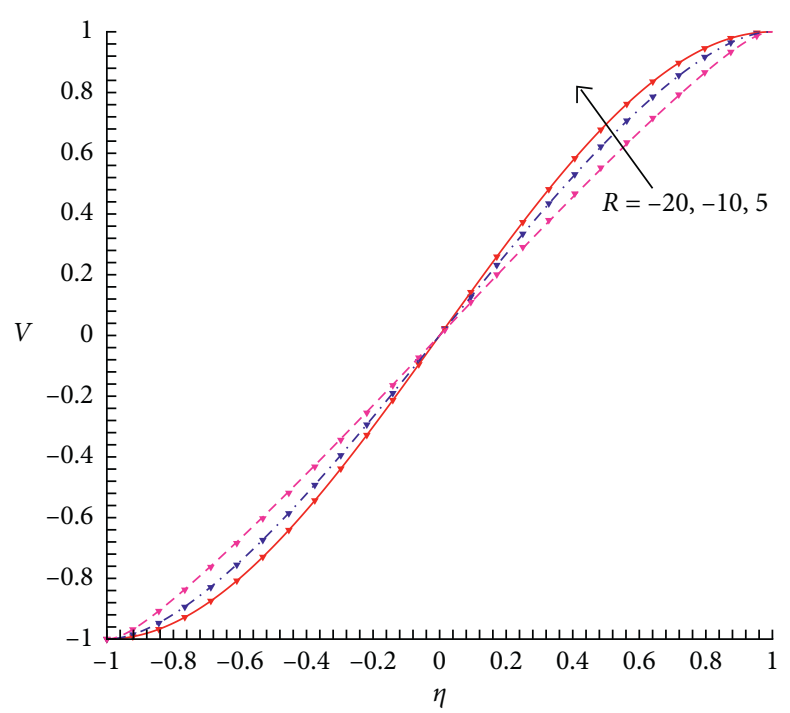

(b)

Figure 4: Comparison of the wavelet-homotopy solutions (line) with the numerical results (diamonds and gradients) of (a) the axial velocity $U$ and (b) the normal velocity $V$ for various $R$ at $\alpha=0$.

computational parameters used here are $c_{0}=-0.08$ for $U$ and $c_{0}=-0.05$ for $V$. The computational order is $M=100$.

\section{Results and Discussion}

As discussed above, investigations of the special case of the fourth-order nonlinear system show excellent computational efficiency of our proposed method. Therefore, we apply it to the common cases of the fourth-order nonlinear system for a range of wall expansion ratios $\alpha$ with different injection or suction Reynolds number $R$. Without loss of generality, the resolution level $j=6$ is used for all the following calculations, but the convergence-control parameter needs to be reasonably selected based on the nonlinear strength of the problem at different $R$ and $\alpha$.

It has been known that the traditional homotopy analysis method is very powerful to give highly accurate solutions of nonlinear problems. Here, we compare the computational capability between the traditional HAM technique and our proposed method, firstly. As moderate physical parameters 
TABle 4: The computational time and relative error of axial velocity $U$ and normal velocity $V$ at different resolution levels.

\begin{tabular}{|c|c|c|c|c|c|}
\hline & Resolution level & $j=3$ & $j=4$ & $j=5$ & $j=6$ \\
\hline$U$ & $\begin{array}{l}\text { CPU time (sec.) } \\
\operatorname{Err}_{M}\end{array}$ & $\begin{array}{c}0.2282 \\
2.2991 \times 10^{-5} \\
\end{array}$ & $\begin{array}{c}0.2735 \\
3.3010 \times 10^{-7} \\
\end{array}$ & $\begin{array}{c}0.3132 \\
4.7763 \times 10^{-9} \\
\end{array}$ & $\begin{array}{c}0.3908 \\
2.7685 \times 10^{-10} \\
\end{array}$ \\
\hline$V$ & $\begin{array}{l}\text { CPU time (sec.) } \\
\operatorname{Err}_{M}\end{array}$ & $\begin{array}{c}0.2407 \\
1.4304 \times 10^{-5} \\
\end{array}$ & $\begin{array}{c}0.2847 \\
1.9613 \times 10^{-8} \\
\end{array}$ & $\begin{array}{c}0.2939 \\
1.7839 \times 10^{-10} \\
\end{array}$ & $\begin{array}{c}0.3857 \\
1.1653 \times 10^{-11} \\
\end{array}$ \\
\hline
\end{tabular}

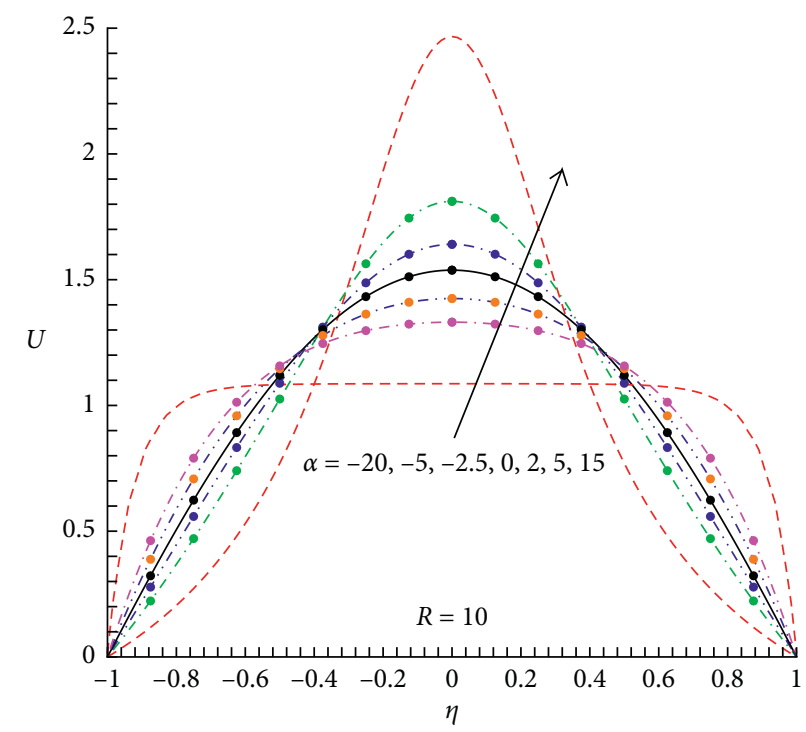

Figure 5: Comparison of the wavelet-homotopy solutions (lines) with the traditional HAM results (circles) over a range of wall expansion ratios $\alpha$ at injection Reynolds number $R=10$.

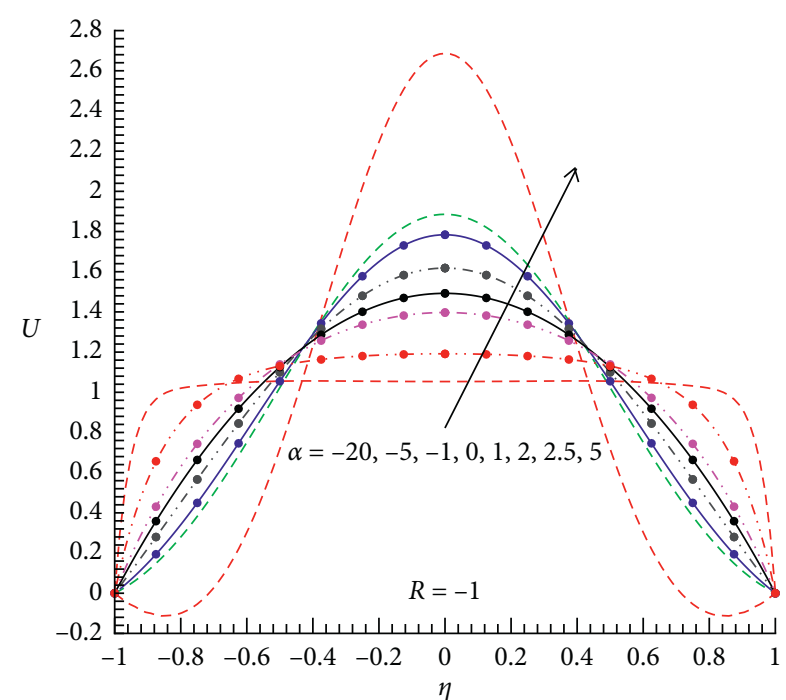

FIGURE 6: Comparison of our wavelet-homotopy solutions (lines) with the HAM results (circles) over a range of wall expansion ratios $\alpha$ at suction Reynolds number $R=-1$.

are prescribed, both approaches give very excellent results. However, our proposed technique is superior to the traditional HAM technique for large physical parameters such as $\alpha=15$ and $\alpha=-20$, as shown in Figures 5 and 6. In these special cases, the homotopy Padé technique [13] has to be used to optimize the HAM results for high-precision solutions.

Tables 5 and 6 give the comparison of CPU times for the maximum values of the axial velocity $U(0)$ by the wavelethomotopy method and HAM technique with different $\alpha$ for $R=10$ and $R=-1$, respectively. Note that the residual errors used here are just an indication to illustrate solution validity. It is seen that our proposed technique has superior computational efficiency over the traditional HAM technique; especially, nonlinearity becomes very strong. For example, in the case of $R=10$ and $\alpha=5$, the HAM technique requires $28,450.3$ seconds to complete the calculation, but the wavelet-homotopy technique only takes 0.6066 second. Note that the resolution level $j=6$ is employed in our computation with the convergent-control parameters being listed in Tables 7 and 8 .

Based on the above analysis, we further obtain the solutions for various physical parameters readily by means of our proposed technique, as shown in Figures 7 and 8. It can be seen that the values of the axial velocity $U$ reach the highest at the middle section of the porous channel which is $\eta=0$, and as the wall expansion ratios, $\alpha$, increase, the maximum value of the axial velocity becomes gradually larger; however, this situation is reversed near the walls. In addition, increasing wall expansion causes more rapid change in the value of the axial velocity from the walls to the middle section of the porous channel, while increasing wall contraction results in a smoother axial velocity curve with a 
TABLE 5: Comparison of CPU times for $U(0)$ given by the wavelet-homotopy method and HAM technique with different $\alpha$ in the case of $R=10$.

\begin{tabular}{|c|c|c|c|c|c|c|}
\hline \multirow{2}{*}{$\alpha$} & \multicolumn{2}{|c|}{$U(0)$} & \multicolumn{2}{|c|}{ Residual error } & \multicolumn{2}{|c|}{ CPU time (s) } \\
\hline & Current & HAM & Current & HAM & Current & HAM \\
\hline 20 & 2.81451 & - & $2.2777 \times 10^{-4}$ & - & 0.7590 & - \\
\hline 15 & 2.46663 & - & $1.9072 \times 10^{-8}$ & - & 0.7298 & - \\
\hline 10 & 2.12775 & - & $1.0711 \times 10^{-9}$ & - & 0.6611 & - \\
\hline 5 & 1.81153 & 1.81161 & $9.9394 \times 10^{-11}$ & $4.5521 \times 10^{-5}$ & 0.6066 & $28,450.3$ \\
\hline 4 & 1.75261 & 1.75264 & $4.8721 \times 10^{-11}$ & $1.3808 \times 10^{-6}$ & 0.5300 & $14,106.4$ \\
\hline 3 & 1.69557 & 1.69558 & $2.2598 \times 10^{-11}$ & $2.9759 \times 10^{-6}$ & 0.5002 & $3,506.92$ \\
\hline 2 & 1.64063 & 1.64064 & $1.0360 \times 10^{-11}$ & $2.3063 \times 10^{-6}$ & 0.5194 & 980.066 \\
\hline 1 & 1.58802 & 1.58803 & $5.3207 \times 10^{-12}$ & $2.4354 \times 10^{-6}$ & 0.4536 & 292.550 \\
\hline 0 & 1.53796 & 1.53796 & $3.4777 \times 10^{-12}$ & $1.3808 \times 10^{-6}$ & 0.3733 & 63.9997 \\
\hline-1 & 1.49059 & 1.49062 & $2.5996 \times 10^{-12}$ & $2.2670 \times 10^{-7}$ & 0.5029 & 32.0802 \\
\hline-2.5 & 1.42503 & 1.42507 & $1.3004 \times 10^{-12}$ & $1.1737 \times 10^{-6}$ & 0.5046 & 32.8491 \\
\hline-5 & 1.33118 & 1.33115 & $8.6448 \times 10^{-12}$ & $3.6382 \times 10^{-6}$ & 0.6230 & 480.474 \\
\hline-10 & 1.19967 & - & $4.7928 \times 10^{-10}$ & - & 0.6400 & - \\
\hline-20 & 1.08636 & - & $6.8731 \times 10^{-8}$ & - & 0.7285 & - \\
\hline
\end{tabular}

TABLE 6: Comparison of CPU times for $U(0)$ given by the wavelet-homotopy method and HAM technique with different $\alpha$ in the case of $R=-1$.

\begin{tabular}{|c|c|c|c|c|c|c|}
\hline \multirow[b]{2}{*}{$\alpha$} & \multicolumn{2}{|c|}{$U(0)$} & \multicolumn{2}{|c|}{ Residual error } & \multicolumn{2}{|c|}{ CPU time (s) } \\
\hline & Current & HAM & Current & HAM & Current & HAM \\
\hline 5 & 2.68605 & - & $5.5546 \times 10^{-10}$ & - & 0.7220 & - \\
\hline 2.5 & 1.88572 & - & $3.0587 \times 10^{-12}$ & - & 0.6875 & - \\
\hline 2 & 1.78380 & 1.78397 & $3.1057 \times 10^{-12}$ & $8.7826 \times 10^{-6}$ & 0.6254 & 974.450 \\
\hline 1 & 1.61802 & 1.61809 & $4.8021 \times 10^{-13}$ & $1.5901 \times 10^{-6}$ & 0.5518 & 37.7529 \\
\hline 0 & 1.49232 & 1.49228 & $2.7859 \times 10^{-15}$ & $4.9944 \times 10^{-6}$ & 0.5630 & 7.22693 \\
\hline-1 & 1.39686 & 1.39663 & $2.1015 \times 10^{-13}$ & $8.4533 \times 10^{-6}$ & 0.6512 & 986.366 \\
\hline-2.5 & 1.29381 & 1.29384 & $4.7729 \times 10^{-12}$ & $8.9765 \times 10^{-6}$ & 0.5960 & $1,682.30$ \\
\hline-5 & 1.19085 & 1.19093 & $1.7206 \times 10^{-10}$ & $1.8075 \times 10^{-6}$ & 0.6622 & $2,181.17$ \\
\hline-10 & 1.10314 & - & $4.2413 \times 10^{-9}$ & - & 0.5935 & - \\
\hline-20 & 1.05330 & - & $7.4941 \times 10^{-9}$ & - & 0.7536 & - \\
\hline
\end{tabular}

TABLE 7: The values of $c_{0}$ used to calculate $U$ for different $R$ and $\alpha$.

\begin{tabular}{|c|c|c|c|c|c|c|}
\hline \multirow{2}{*}{$U$} & \multicolumn{2}{|c|}{$R=10$} & \multicolumn{2}{|c|}{$R=50$} & \multicolumn{2}{|c|}{$R=200$} \\
\hline & $\alpha$ & $c_{0}$ & $\alpha$ & $c_{0}$ & $\alpha$ & $c_{0}$ \\
\hline & 20 & -0.03 & 20 & -0.05 & 100 & -0.005 \\
\hline & 15 & -0.055 & 10 & -0.06 & 50 & -0.02 \\
\hline & 10 & -0.07 & 5 & -0.06 & 20 & -0.03 \\
\hline & $0 \sim 5$ & -0.08 & 0 & -0.06 & 0 & -0.05 \\
\hline & $-1 \sim-5$ & -0.08 & -5 & -0.06 & -20 & -0.05 \\
\hline & -10 & -0.08 & -20 & -0.06 & -50 & -0.06 \\
\hline & -20 & -0.09 & -50 & -0.06 & -100 & -0.02 \\
\hline & \multicolumn{2}{|c|}{$R=-1$} & \multicolumn{2}{|c|}{$R=-5$} & \multicolumn{2}{|c|}{$R=-10$} \\
\hline & $\alpha$ & $c_{0}$ & $\alpha$ & $c_{0}$ & $\alpha$ & $c_{0}$ \\
\hline & 5 & -0.16 & 2.5 & -0.5 & 1 & -0.16 \\
\hline & 2.5 & -0.14 & 2 & -0.2 & 0 & -0.08 \\
\hline & 2 & -0.13 & 1 & -0.08 & -1 & -0.08 \\
\hline & 1 & -0.11 & 0 & -0.08 & -5 & -0.08 \\
\hline & $0,-1$ & -0.08 & -1 & -0.08 & -20 & -0.07 \\
\hline & -2.5 & -0.02 & -5 & -0.08 & & \\
\hline & -5 & -0.013 & -20 & -0.08 & & \\
\hline & $-10,-20$ & -0.01 & & & & \\
\hline
\end{tabular}

wider range of plateaus. What is noteworthy is that flow reversal happens near the walls at $R=-5, \alpha=2$ and 2.5 , and $R=-10, \quad \alpha=1$ cases, which is the result of the rapid expansion of the wall creating a suction area near the wall, so the fluid is forced to move in the direction of the wall to occupy the space created by the wall expansion process. This 
TABLE 8: The values of $c_{0}$ used to calculate $V$ for different $R$ and $\alpha$.

\begin{tabular}{|c|c|c|c|c|c|c|c|c|}
\hline \multirow{2}{*}{$V$} & \multicolumn{2}{|c|}{$R=5$} & \multicolumn{2}{|c|}{$R=20$} & \multicolumn{2}{|c|}{$R=-1$} & \multicolumn{2}{|c|}{$R=-5$} \\
\hline & $\alpha$ & $c_{0}$ & $\alpha$ & $c_{0}$ & $\alpha$ & $c_{0}$ & $\alpha$ & $c_{0}$ \\
\hline & 15 & -0.125 & 20 & -0.05 & 5 & -0.08 & 2.5 & -0.5 \\
\hline & 5 & -0.05 & 5 & -0.06 & 2 & -0.08 & 1 & -0.18 \\
\hline & 0 & -0.05 & 0 & -0.07 & 1 & -0.15 & 0 & -0.12 \\
\hline & -5 & -0.05 & -5 & -0.08 & 0 & -0.1 & -5 & -0.1 \\
\hline & -20 & -0.2 & -20 & -0.11 & -5 & -0.08 & -20 & -0.08 \\
\hline & & & & & -20 & -0.06 & & \\
\hline
\end{tabular}

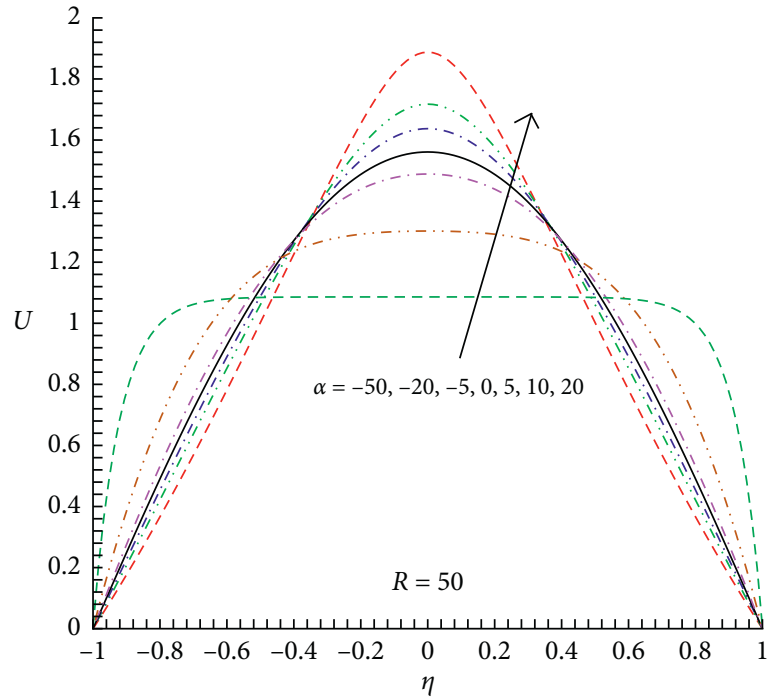

(a)

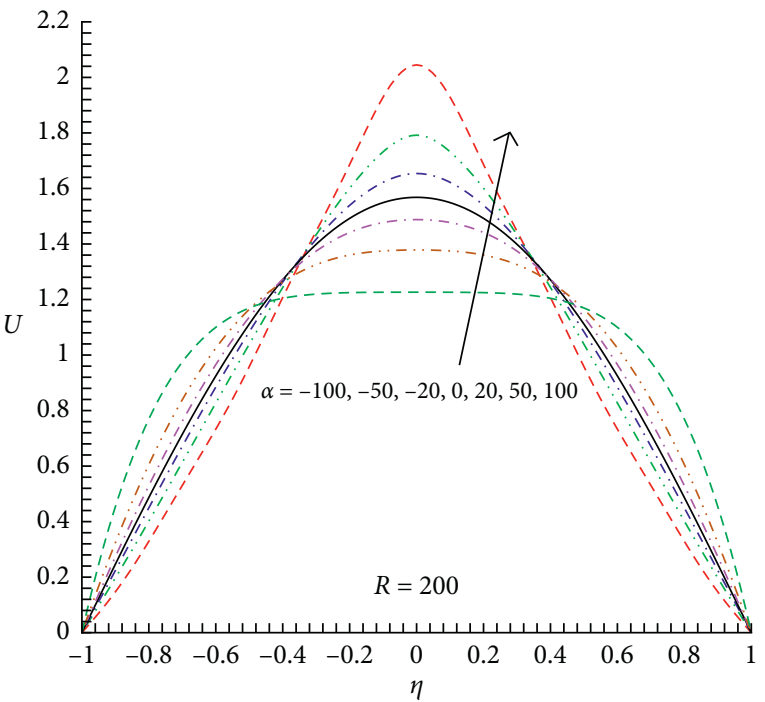

(b)

Figure 7: The values of axial velocity $U$ obtained by the wavelet-homotopy method in different wall expansion ratios $\alpha$ at (a) $R=50$ and (b) $R=200$.

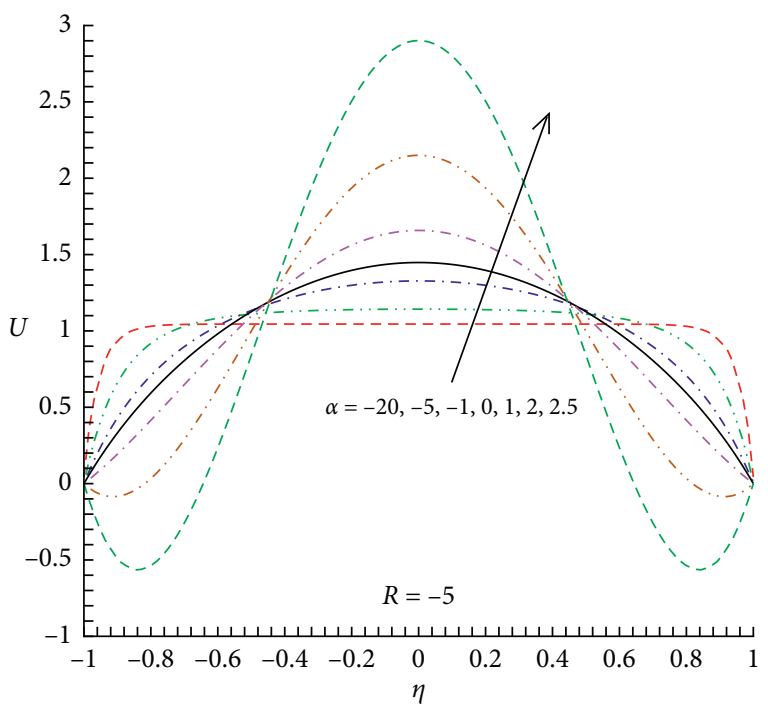

(a)

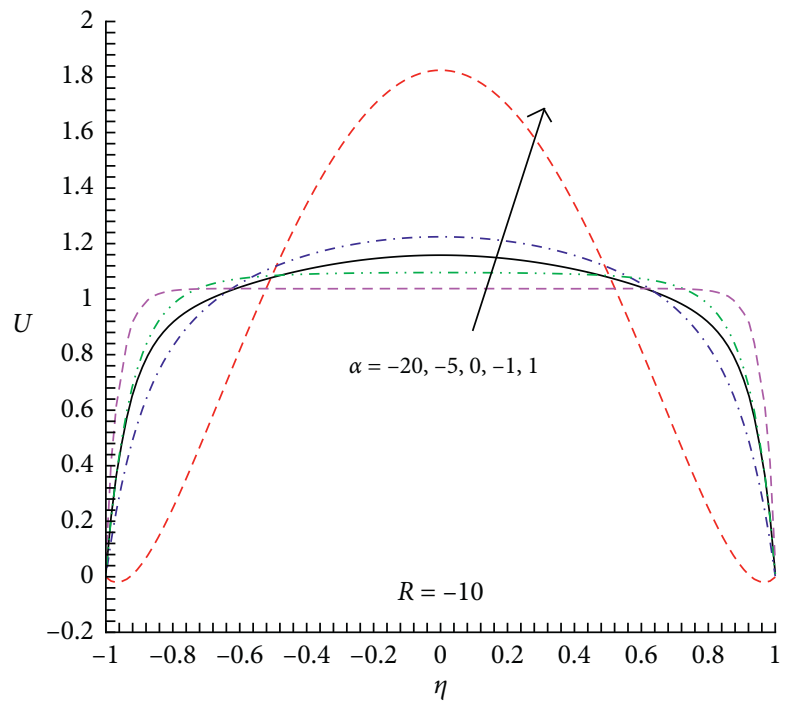

(b)

FIgURE 8: The values of axial velocity $U$ obtained by the wavelet-homotopy method in different wall expansion ratios $\alpha$ at (a) $R=-5$ and (b) $R=-10$. 
is also consistent with the findings by Dauenhauer and Majdalani [12].

\section{Conclusions}

In this paper, the wavelet-homotopy method has been developed to obtain highly accurate solutions of fourth-order nonlinear boundary value problems describing fluid flows in the porous channel with moving walls. By far, now, homotopy-wavelet technique has been investigated for this kind of problems. The full solution procedure has been given in detail. We have performed wavelet approximation on the homogeneous Newman boundary condition by modifying the Coiflet wavelet basis function. The efficiency and accuracy of the Coiflet wavelet method have been verified by an example of a linear two-dimensional problem. Furthermore, the application of our homotopy-based Coiflet wavelet method to nonlinear problems has been carried out. By comparing with the results of the traditional homotopy analysis method, the reliability and efficiency of our Coiflet wavelet-homotopy method in solving strong nonlinear problems are further explained. The solutions of classic problems with liquid flow in porous channels for various physical parameters have been obtained readily by our proposed method. It is expected that this technique is expected to give accurate solutions for more complicated problems with strong nonlinearity.

\section{Nomenclature}

$\alpha: \quad$ Wall expansion and extraction ratio

$\Gamma, \bar{\Gamma}: \quad$ Generalized connection coefficient

A, B: Connection coefficient matrix in the waveletGalerkin deformation equation

$\mathscr{L}: \quad$ Auxiliary linear operator

$\mathcal{N}: \quad$ Nonlinear operator

$\phi$ : $\quad$ Mapping function of $F$ in HAM

$\varphi$ : $\quad$ Coiflet wavelet basis

$c_{0}$ : Convergence-control parameter

$\operatorname{Err}_{M}$ : Relative error

ErrSQ: Average square error

$F$ : Dimensionless stream function

$F_{0}$ : Initial guess

$h$ : Coiflet wavelet basis under Neumann types of boundary conditions

$M_{1}$ : First-order vanishing moment of the Coiflet wavelet

$N$ : Vanishing moment of the Coiflet wavelet

p: $\quad$ Coefficient in modified Coiflet wavelet basis

$P$ : $\quad$ Coefficient matrix in modified Coiflet wavelet basis

q: $\quad$ Embedding parameter in HAM

$R: \quad$ Reynolds number

ResErr: Residual error

$U, V: \quad$ Axial velocity and normal velocity.

\section{Data Availability}

The data used to support the findings of this study are included within the article.

\section{Conflicts of Interest}

The authors declare that there are no conflicts of interest regarding the publication of this paper.

\section{Acknowledgments}

This work was partially supported by the National Natural Science Foundation of China (Grant no. 11872241).

\section{References}

[1] A. S. Berman, "Laminar flow in channels with porous walls," Journal of Applied Physics, vol. 24, no. 9, pp. 1232-1235, 1953.

[2] M. Morduchow, "On laminar flow through a channel or tube with injection: application of method of averages," Quarterly of Applied Mathematics, vol. 14, no. 4, pp. 361-368, 1957.

[3] I. Proudman, "An example of steady laminar flow at large Reynolds number," Journal of Fluid Mechanics, vol. 9, no. 4, pp. 593-602, 1960.

[4] T. Wah, "Laminar flow in a uniformly porous channel," Aeronautical Quarterly, vol. 15, no. 3, pp. 299-310, 1964.

[5] J. R. Sellars, "Laminar flow in channels with porous walls at high suction Reynolds numbers," Journal of Applied Physics, vol. 26, no. 4, pp. 489-490, 1955.

[6] G. Taylor, "Fluid flow in regions bounded by porous surfaces," Proceedings of the Royal Society of London. Series A, Mathematical and Physical Sciences, vol. 234, no. 1199, pp. 456-475, 1956.

[7] S. W. Yuan, "Further investigation of laminar flow in channels with porous walls," Journal of Applied Physics, vol. 27, no. 3, pp. 267-269, 1956.

[8] R. M. Terrill, "Laminar flow in a uniformly porous channel with large injection," Aeronautical Quarterly, vol. 16, no. 4, pp. 323-332, 1965.

[9] F. M. White Jr., "Laminar flow in a uniformly porous tube," Journal of Applied Mechanics, vol. 29, no. 1, pp. 201-204, 1962.

[10] J. F. Brady and A. Acrivos, "Steady flow in a channel or tube with an accelerating surface velocity. An exact solution to the Navier-Stokes equations with reverse flow," Journal of Fluid Mechanics, vol. 112, no. 1, pp. 127-150, 1981.

[11] E. B. B. Watson, W. H. H. Banks, M. B. Zaturska, and P. G. Drazin, "On transition to chaos in two-dimensional channel flow symmetrically driven by accelerating walls," Journal of Fluid Mechanics, vol. 212, no. 1, pp. 451-485, 1990.

[12] E. C. Dauenhauer and J. Majdalani, "Exact self-similarity solution of the Navier-Stokes equations for a porous channel with orthogonally moving walls," Physics of Fluids, vol. 15, no. 6, pp. 1485-1495, 2003.

[13] H. Xu, Z. L. Lin, S. J. Liao, J. Z. Wu, and J. Majdalani, "Homotopy based solutions of the Navier-Stokes equations for a porous channel with orthogonally moving walls," Physics of Fluids, vol. 22, no. 5, Article ID 053601, 2010.

[14] M. Gholinia, K. Hosseinzadeh, H. Mehrzadi, D. D. Ganji, and A. A. Ranjbar, "Investigation of MHD Eyring-Powell fluid flow over a rotating disk under effect of homogeneous-heterogeneous reactions," Case Studies in Thermal Engineering, vol. 13, Article ID 100356, 2019.

[15] Kh. Hosseinzadeh, A. Asadi, A. R. Mogharrebi, J. Khalesi, S. Mousavisanib, and D. D. Ganji, "Entropy generation analysis of $\left(\mathrm{CH}_{2} \mathrm{OH}\right)_{2}$ containing CNTs nanofluid flow under effect of MHD and thermal radiation," Case Studies in Thermal Engineering, vol. 14, Article ID 100482, 2019. 
[16] M. R. Zangooee, K. Hosseinzadeh, and D. D. Ganji, "Hydrothermal analysis of MHD nanofluid $\left(\mathrm{TiO}_{2}-\mathrm{GO}\right)$ flow between two radiative stretchable rotating disks using AGM," Case Studies in Thermal Engineering, vol. 14, Article ID 100460, 2019.

[17] R. Derakhshan, A. Shojaei, K. Hosseinzadeh, M. Nimafar, and D. D. Ganji, "Hydrothermal analysis of magneto hydrodynamic nanofluid flow between two parallel by AGM," Case Studies in Thermal Engineering, vol. 14, Article ID 100439, 2019.

[18] K. Hosseinzadeh, A. J. Amiri, S. S. Ardahaie, and D. D. Ganji, "Effect of variable lorentz forces on nanofluid flow in movable parallel plates utilizing analytical method," Case Studies in Thermal Engineering, vol. 10, pp. 595-610, 2017.

[19] K. Hosseinzadeh, A. R. Mogharrebi, A. Asadi, M. Paikar, D. D. Ganji, and D. D. Ganji, "Effect of fin and hybrid nanoparticles on solid process in hexagonal triplex latent heat thermal energy storage system," Journal of Molecular Liquids, vol. 300, Article ID 112347, 2020.

[20] M. Gholinia, S. Gholinia, K. Hosseinzadeh, and D. D. Ganji, "Investigation on ethylene glycol nano fluid flow over a vertical permeable circular cylinder under effect of magnetic field," Results in Physics, vol. 9, pp. 1525-1533, 2018.

[21] K. Hosseinzadeh, M. Gholinia, B. Jafari, A. Ghanbarpour, H. Olfian, and D. D. Ganji, "Nonlinear thermal radiation and chemical reaction effects on Maxwell fluid flow with convectively heated plate in a porous medium," Heat TransferAsian Research, vol. 48, no. 2, pp. 744-759, 2019.

[22] T. Liu, "A wavelet multiscale-homotopy method for the parameter identification problem of partial differential equations," Computers \& Mathematics with Applications, vol. 71, no. 7, pp. 1519-1523, 2016.

[23] T. Liu, "Reconstruction of a permeability field with the wavelet multiscale-homotopy method for a nonlinear convection-diffusion equation," Applied Mathematics and Computation, vol. 275, pp. 432-437, 2016.

[24] T. Liu, "A multigrid-homotopy method for nonlinear inverse problems," Computers \& Mathematics with Applications, vol. 79, no. 6, pp. 1706-1717, 2020.

[25] T. Liu and S. Liu, "Identification of diffusion parameters in a non-linear convection-diffusion equation using adaptive homotopy perturbation method," Inverse Problems in Science and Engineering, vol. 26, no. 4, pp. 464-478, 2018.

[26] S. J. Liao, Homotopy Analysis Method in Nonlinear Differential Equations, Springer, Berlin, Germany, 2012.

[27] A. Shojaei, A. J. Amiri, S. S. Ardahaie, K. Hosseinzadeh, and D. D. Ganji, "Hydrothermal analysis of non-Newtonian second grade fluid flow on radiative stretching cylinder with Soret and Dufour effects," Case Studies in Thermal Engineering, vol. 13, Article ID 100384, 2019.

[28] X. Zhong and S. Liao, "On the limiting Stokes wave of extreme height in arbitrary water depth," Journal of Fluid Mechanics, vol. 843, pp. 653-679, 2018.

[29] I. Daubechies, "Orthonormal bases of compactly supported wavelets," Communications on Pure and Applied Mathematics, vol. 41, no. 7, pp. 909-996, 1988.

[30] S. J. Huang and C. T. Hsieh, "Coiflet wavelet transform applied to inspect power system disturbance-generated signals," IEEE Transactions on Aerospace and Electronic Systems, vol. 38, no. 1, pp. 204-210, 2002.

[31] Y. H. Zhou and J. Z. Wang, "Generalized Gaussian integral method for calculations of scaling function transform of wavelets and its applications," Acta Mathematica Scientia, vol. 19, no. 3, pp. 293-300, 1999, in Chinese.
[32] Y. H. Zhou, X. M. Wang, J. Z. Wang, and X. J. Liu, “A wavelet numerical method for solving nonlinear fractional vibration, diffusion and wave equations," Computer Modeling in Engineering and Sciences, vol. 77, no. 2, pp. 137-160, 2011.

[33] X. Liu, Y. Zhou, X. Wang, and J. Wang, "A wavelet method for solving a class of nonlinear boundary value problems," Communications in Nonlinear Science and Numerical Simulation, vol. 18, no. 8, pp. 1939-1948, 2013.

[34] X. Liu, Y. Zhou, L. Zhang, and J. Wang, "Wavelet solutions of Burgers' equation with high Reynolds numbers," Science China Technological Sciences, vol. 57, no. 7, pp. 1285-1292, 2014.

[35] Z. Yang and S. Liao, "A HAM-based wavelet approach for nonlinear ordinary differential equations," Communications in Nonlinear Science and Numerical Simulation, vol. 48, pp. 439-453, 2017.

[36] Z. Yang and S. Liao, "A HAM-based wavelet approach for nonlinear partial differential equations: two dimensional Bratu problem as an application," Communications in Nonlinear Science and Numerical Simulation, vol. 53, pp. 249-262, 2017.

[37] Q. Yu, H. Xu, and S. Liao, "Nonlinear analysis for extreme large bending deflection of a rectangular plate on non-uniform elastic foundations," Applied Mathematical Modelling, vol. 61, pp. 316-340, 2018.

[38] Q. Yu, H. Xu, S. Liao, and Z. Yang, "A novel homotopywavelet approach for solving stream function-vorticity formulation of Navier-Stokes equations," Communications in Nonlinear Science and Numerical Simulation, vol. 67, pp. 124-151, 2019.

[39] J. Z. Wang, Generalized theory and arithmetic of orthogonal wavelets and applications to researches of mechanics including piezoelectric smart structures, Ph.D. thesis, Lanzhou University, Lanzhou, China, 2001, in Chinese.

[40] X. J. Liu, A wavelet method for uniformly solving nonlinear problems and its application to quantitative research on flexible structures with large deformation, Ph.D. thesis, Lanzhou University, Lanzhou, China, 2014, in Chinese.

[41] Q. Yu, H. Xu, and S. Liao, "Analysis of mixed convection flow in an inclined lid-driven enclosure with Buongiorno's nanofluid model," International Journal of Heat and Mass Transfer, vol. 126, pp. 221-236, 2018.

[42] Q. Yu, H. Xu, and S. J. Liao, "Coiflets solutions for Föppl-von Kármán equations governing large deflection of a thin flat plate by a novel wavelet-homotopy approach," Numerical Algorithms, vol. 79, no. 4, pp. 993-1020, 2018.

[43] Q. Yu and H. Xu, "Novel wavelet-homotopy Galerkin technique for analysis of lid-driven cavity flow and heat transfer with non-uniform boundary conditions," Applied Mathematics and Mechanics, vol. 39, no. 12, pp. 1691-1718, 2018. 